\title{
INITIAL GROWTH OF Bauhinia variegata TREES UNDER DIFFERENT COLORED SHADE NETS AND LIGHT CONDITIONS ${ }^{1}$
}

\author{
Renata Bachin Mazzini-Guedes² e Kathia Fernandes Lopes Pivetta ${ }^{3}$
}

\begin{abstract}
Bauhinia variegata and B. variegata var. candida, commonly known as orchid trees, are small sized trees widely used for urban forestry and landscaping. Adult plants grow under full sun; in Brazil, however, seedlings are generally cultivated in commercial nurseries under natural half-shading. The objective of this study was to evaluate the influence of different colored shade nets and light conditions on the initial growth of $B$. variegata and $B$. variegata var. candida. The influence of six light conditions (red net with $50 \%$ shading; blue net with $50 \%$ shading; black net with $70 \%$ shading; black net with $50 \%$ shading; black net with $30 \%$ shading; and full sun) on the initial growth of B. variegata and B. variegata var. candida were evaluated along 160 days, and growth relationships were calculated. Seedlings showed more efficiency on the use of photoassimilated compounds when grown under full sun. Such condition is the most appropriate for seedling production of $B$. variegata and B. variegata var. candida, contradicting what has been performed in practice.
\end{abstract}

Keywords: Orchid tree; Shading; Ornamental tree.

\section{CRESCIMENTO INICIAL DE MUDAS DE Bauhinia variegata SOB DIFERENTES TELAS COLORIDAS E CONDIÇÕES DE LUMINOSIDADE}

RESUMO - Bauhinia variegata e B. variegata var. candida, popularmente conhecidas como pata-de-vaca, são árvores de porte baixo muito usadas na arborização urbana e no paisagismo. Plantas adultas crescem sob sol pleno; no Brasil, no entanto, mudas são geralmente cultivadas em viveiros comerciais sob meiasombra. O objetivo deste trabalho foi avaliar a influência de diferentes telas coloridas e condições de luminosidade no crescimento inicial de mudas de B. variegata e B. variegata var. candida. A influência de seis condições de luminosidade (tela vermelha com 50\% de sombreamento, tela azul com 50\% de sombreamento, tela preta com $70 \%$ de sombreamento, tela preta com $50 \%$ de sombreamento, tela preta com $30 \%$ de sombreamento e a sol pleno) no crescimento inicial das mudas foi avaliada ao longo de 160 dias, e relações de crescimento foram calculadas. As mudas foram mais eficientes no uso dos fotoassimilados quando cultivadas a sol pleno. Essa condição é a mais apropriada para a produção de mudas de B. variegata e B. variegata var. candida, contrariando o que vem sendo feito na prática.

Keywords: Pata-de-vaca; Sombreamento; Árvore ornamental.

\footnotetext{
${ }^{1}$ Recebido em 15.11.2013 aceito para publicação em 10.11.2014

${ }^{2}$ Doutorado em Agronomia (Produção Vegetal), UNESP Univ Estadual Paulista 'Júlio de Mesquita Filho', Faculdade de Ciências Agrárias e Veterinárias (FCAV), Departamento de Produção Vegetal - Jaboticabal, SP - Brasil. E-mail: <remazzini@yahoo.com.br> . ${ }^{3}$ UNESP Univ Estadual Paulista 'Júlio de Mesquita Filho', Faculdade de Ciências Agrárias e Veterinárias, FCAV, Departamento de Produção Vegetal, Jaboticabal, SP - Brasil. E-mail: <kathia@fcav.unesp.br>.
} 


\section{INTRODUCTION}

Bauhinia variegata L., belonging to the family Fabaceae and native to South China (MAK et al., 2008), is a small sized tree widely used for urban forestry and landscaping. According to Aguiar and Figliolia (1996), it is probably the most used Bauhinia species for ornamental purposes. It is a fast growing plant (MATHUR; MUKUNTHAKUMAR, 1992), reaching around $8 \mathrm{~m}$ height and $8 \mathrm{~m}$ crown diameter, that flowers mainly from December to April (LAU et al., 2005), producing pink flowers of different tones (GLOBAL..., 2003). B. variegata var. candida (Aiton) Voigt is also a small sized tree, reaching around $6 \mathrm{~m}$ height, that flowers mainly from June to December (RORIZ, 2000) and produces white flowers (MAK et al., 2008). Both are commonly known as orchid trees and usually grow under full sun (RORIZ, 2000; GLOBAL..., 2003).

The sunlight, according to its duration, intensity, and quality, is essential for plant growth and flower production (SHAHAK et al., 2004a). It affects several physiological processes, such as photosynthesis, that is primarily responsible for the accumulation of plant dry matter (BENINCASA, 2003). According to the species and its habitat, a certain light amount can either limit or optimize its development (MELEIRO; GRAZIANO, 2007).

Black nets, that partially filter the sunlight, and colored nets, that modify it, have been widely used for agricultural purposes (SHAHAK et al., 2004a; MEIRELLES et al., 2007). They provide more shading for plants and, also, protection against wind, hail, pests, and other extreme environmental conditions (LOBOS et al., 2013). Furthermore, their application improves the internal climatic conditions, increasing air humidity and reducing temperature (SHAHAK et al., 2004a,b; LOBOS et al., 2013). While black nets transmit a scattered and uniform light, therefore maintaining light quality, colored ones modify both light quantity and quality (OREN-SHAMIR et al., 2001). Blue nets, for instance, transmit green and blue lights (400-540 nm) absorbing the red light (550-660 nm), while red nets transmit light at $590 \mathrm{~nm}$, i.e., the light spectrum region that is best absorbed by plants resulting, therefore, in higher photosynthesis rate (SHAHAK et al., 2004b).

In Brazil, tree seedlings are generally cultivated, for commercial purposes, under half-shading. Such condition, promoted by either black or colored nets, usually avoid high light and temperature (LI; SYVERTSEN, 2006), what could be detrimental for sunsensitive seedlings. As studies on the light or shading influence on Bauhinia variegata and B. variegata var. candida growth were not found, the objective of this study was to evaluate the influence of different colored nets and light conditions on the initial growth of those species.

\section{MATERIALS AND METHODS}

Mature brown seed pods, closed or partially open, were harvested in September 2010 from 20 year old $B$. variegata and $B$. variegata var. candida trees cultivated at the College of Agricultural and Veterinary Sciences of the State University of São Paulo (FCAV/ UNESP), located in the municipality of Jaboticabal, Brazil (21 $15^{\circ} 22^{\prime \prime} \mathrm{S}$ and $48^{\circ} 18^{\prime} 58^{\prime \prime} \mathrm{W}$, at $590 \mathrm{~m}$ altitude). According to the Köppen classification, the climate regime in Jaboticabal is subtropical with dry winters and humid summers (Cwa). The experiment was implemented at the Experimental Nursery of Ornamental and Forest Plants of the FCAV/UNESP.

Immediately after harvesting, the seeds were cleaned and kept in paper bags at room temperature for about three months. Seeds were then sown in Plantmax substrate (Eucatex ${ }^{\circledR}$ ) in December 2010 and, after 20 days, seedlings were transplanted to black plastic bags of $2 \mathrm{~L}$ capacity that contained soil and cattle manure (3:1, v:v). The soil, collected at $0.0-0.2 \mathrm{~m}$ depth at the campus area, was classified as Oxisol. Soil plus cattle manure analysis was performed according to Raij et al. (2001) at the Laboratory of Soil Analysis of the FCAV/ UNESP. As it was considered appropriate (data not shown), there was no additional fertilization throughout the experimental period.

In January 2011, seedlings were submitted to different light conditions, which consisted of six treatments: red net with 50\% shading (red net 50\%), blue net with $50 \%$ shading (blue net 50\%), black net with 70\% shading (black net 70\%), black net with 50\% shading (black net 50\%), black net with 30\% shading (black net 30\%), and full sun. The structure for each treatment was built as a tunnel and measured $6.0 \mathrm{~m}$ length, $1.5 \mathrm{~m}$ width and $1.8 \mathrm{~m}$ height. The tunnels, which were entirely coated with each net, were placed $2 \mathrm{~m}$ apart from each other, according to the sun eastwest direction, so that there was no additional shading that could influence plant growth. The average

Revista Árvore, Viçosa-MG, v.38, n.6, p.1133-1145, 2014 
photosynthetically active radiation (PAR) during the experimental period for each treatment was 54.0, 70.3, 38.7, 75.1, 104.7, and $143.8 \mathrm{~W} \mathrm{~m}^{-2}$ for the red net $50 \%$, blue net $50 \%$, black net $70 \%$, black net $50 \%$, black net $30 \%$, and full sun, respectively. Average minimum, mean, and maximum air temperature were, respectively, 17.4, 22.1, and $29.1^{\circ} \mathrm{C}$; average air humidity was $77.4 \%$; air temperature and humidity were monitored at the experiment location. Irrigation was performed by a dripping system that remained on along four hours in the afternoons, three times per week. Each pot had an individual dripper that released $400 \mathrm{~mL}$ water during those four hours, therefore, 1.2 L per week. Signals of water deficit or symptoms of pests and diseases were not observed.

The experimental design was entirely randomized with six treatments and four replications (one initial evaluation and four evaluations along the experimental period). Each replication consisted of four plants, resulting in 96 plants of each species.

Seedling growth was evaluated by collecting one plant per replication, per treatment, at 40, 80, 120, and 160 days after the beginning of the experiment. Evaluated characteristics were: stem length, stem diameter, leaf number, leaf area (LI-3100 area meter, LI-Cor ${ }^{\circledR}$, Lincoln, NE, USA), and dry matter of roots and aerial part, which was divided into dry matter of stem and leaves. Data were submitted to variance analysis followed by the Tukey test at 5\% significance level. The polynomial regression was also applied for evaluation of the studied variables along time.

Several growth relationships, which express plant growth, were also calculated as described by Benincasa (2003): absolute growth rate of roots (AGR), that determines root growth speed, in g day $^{-1}$; leaf area ratio (LAR), that expresses the photosynthetically active leaf area and defines the leaf area that has been used by the plant to produce $1 \mathrm{~g}$ of dry matter, in $\mathrm{cm}^{2} \mathrm{~g}^{-1}$; and leaf weight ratio (LWR), that expresses the dry matter fraction that is not exported to other plant parts and remains in the leaves.

\section{RESULTS}

For B. variegata, stem length was the only variable that was statistically different among treatments for all the evaluated periods (Table 1). At the first evaluation (at 40 days), all net treatments showed similar results, with the exception of the black net 30\%, which, in turn, was statistically equal to both blue net $50 \%$ and full sun treatments.

The influence of the treatments on stem length varied along the experimental period. However, at the last evaluation (at 160 days), the black net $70 \%$ stood out for promoting the longest stem length of the experiment, feature that was maintained from the previous evaluation, performed at 120 days. These plants produced stems that were around $10 \mathrm{~cm}$ longer than plants grown under the red net 50\%, that presented the second highest value (Table 1). Plants cultivated under full sun showed a regular growth throughout the experimental period, with shorter stems up to the last evaluation, when it was statistically different from the others.

The variables stem diameter and leaf number did not present significant differences among treatments for all evaluation periods (Table 1).

Regarding leaf area, there were statistical differences among treatments (Table 1). At the first evaluation (at 40 days), all net treatments had statistically equal values. However, at that time, the black net $30 \%$ was also similar to full sun, which presented the lowest result. During the experimental period, these results varied and, at 160 days, the red net 50\%, black net $70 \%$, and black net $50 \%$ treatments stood out, but only the black net $70 \%$ was different from the other treatments, i.e., the red net $50 \%$ and black net $50 \%$ were also statistically similar to the others.

Dry matter variables showed similar results throughout the experiment, with the exception of dry matter of aerial part and leaves, which presented a significant difference among treatments at 40 days (Table 1). At this evaluation, all plants cultivated under any kind of net had similar results; however, for dry matter of aerial part, the red net 50\% was the only treatment that was different from full sun. For dry matter of leaves, the red net $50 \%$, blue net $50 \%$, and black net $50 \%$ had different values from the full sun treatment, reaching the best results.

Analysis of $B$. variegata var. candida data showed that both species had similar responses to the shading conditions (Table 2). However, the last evaluation (at 160 days) indicated that, unlike what was found for $B$. variegata, the red net $50 \%$, blue net $50 \%$, black net $50 \%$, and black net $70 \%$ did influence $B$. variegata var. candida stem length. Although the black net $70 \%$

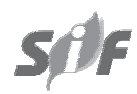

Revista Árvore, Viçosa-MG, v.38, n.6, p.1133-1145, 2014 
Table 1 - Stem length (SL), stem diameter (SD), leaf number (LN), leaf area (LA), and dry matter of roots (DMR), aerial part (DMAP), stem (DMS), and leaves (DML) of Bauhinia variegata seedlings cultivated under different light conditions along 160 days (2011).

Tabela 1 - Comprimento de caule (SL), diâmetro de caule (SD), número de folhas (LN), área foliar (LA) e matéria seca de raízes (DMR), parte aérea (DMAP), caule (DMS) e folhas (DML) de mudas de Bauhinia variegata cultivadas sob diferentes condições de luminosidade, ao longo de 160 dias (2011).

\begin{tabular}{|c|c|c|c|c|c|c|c|c|}
\hline \multicolumn{9}{|c|}{ Bauhinia variegata } \\
\hline Light conditions & $\mathrm{SL}(\mathrm{cm})$ & $\mathrm{SD}(\mathrm{cm})$ & $\mathrm{LN}$ & $\mathrm{LA}\left(\mathrm{cm}^{2}\right)$ & DMR(g) & DMAP(g) & DMS (g) & DML(g) \\
\hline \multicolumn{9}{|c|}{40 days $^{1}$} \\
\hline Red net 50\% & $49.1 \mathrm{a}$ & $0.53 \mathrm{a}$ & $12.8 \mathrm{a}$ & $334.33 \mathrm{a}$ & $1.11 \mathrm{a}$ & $1.96 \mathrm{a}$ & $0.89 \mathrm{a}$ & $1.07 \mathrm{a}$ \\
\hline Blue net $50 \%$ & $47.0 \mathrm{ab}$ & 0.68 a & 12.8 a & $348.87 \mathrm{a}$ & $1.11 \mathrm{a}$ & $1.89 \mathrm{ab}$ & $0.88 \mathrm{a}$ & $1.01 \mathrm{a}$ \\
\hline Black net $70 \%$ & $51.8 \mathrm{a}$ & $0.55 \mathrm{a}$ & $13.0 \mathrm{a}$ & $352.05 \mathrm{a}$ & 0.34 a & $1.47 \mathrm{ab}$ & $0.68 \mathrm{a}$ & $0.79 \mathrm{ab}$ \\
\hline Black net $50 \%$ & 49.9 a & 0.65 a & 13.8 a & 353.16 a & 0.59 a & $1.90 \mathrm{ab}$ & 0.88 a & $1.03 \mathrm{a}$ \\
\hline Black net $30 \%$ & 37.4 bc & $0.44 \mathrm{a}$ & $11.3 \mathrm{a}$ & $221.78 \mathrm{ab}$ & $0.74 \mathrm{a}$ & $1.22 \mathrm{ab}$ & 0.53 a & $0.70 \mathrm{ab}$ \\
\hline Full sun & $31.0 \mathrm{c}$ & $0.54 \mathrm{a}$ & $10.5 \mathrm{a}$ & $122.10 \mathrm{~b}$ & $0.71 \mathrm{a}$ & $0.93 \mathrm{~b}$ & $0.50 \mathrm{a}$ & $0.43 \mathrm{~b}$ \\
\hline $\mathrm{CV} \%$ & 11.6 & 20.5 & 15.8 & 24.2 & 57.3 & 28.4 & 29.1 & 29.3 \\
\hline \multicolumn{9}{|c|}{80 days $^{1}$} \\
\hline Red net 50\% & $61.4 \mathrm{a}$ & $0.91 \mathrm{a}$ & $13.5 \mathrm{a}$ & $309.72 \mathrm{ab}$ & $2.96 \mathrm{a}$ & $4.33 \mathrm{a}$ & $2.48 \mathrm{a}$ & $1.85 \mathrm{a}$ \\
\hline Blue net $50 \%$ & $61.4 \mathrm{a}$ & $0.84 \mathrm{a}$ & $14.0 \mathrm{a}$ & $267.93 \mathrm{ab}$ & 2.79 a & 4.52 a & $2.30 \mathrm{a}$ & $2.21 \mathrm{a}$ \\
\hline Black net $70 \%$ & 70.9 a & 0.89 a & 16.0 a & 354.62 a & $1.87 \mathrm{a}$ & 4.18 a & 2.08 a & $2.10 \mathrm{a}$ \\
\hline Black net $50 \%$ & $53.9 \mathrm{ab}$ & 0.94 a & $13.0 \mathrm{a}$ & 200.50 bc & 2.99 a & $4.28 \mathrm{a}$ & $2.46 \mathrm{a}$ & $1.82 \mathrm{a}$ \\
\hline Black net $30 \%$ & 63.9 a & 0.99 a & 13.8 a & 317.76 a & $4.05 \mathrm{a}$ & $5.81 \mathrm{a}$ & 3.23 a & $2.58 \mathrm{a}$ \\
\hline Full sun & $43.9 \mathrm{~b}$ & 0.86 a & $14.3 \mathrm{a}$ & $149.48 \mathrm{c}$ & $3.43 \mathrm{a}$ & $3.00 \mathrm{a}$ & $1.64 \mathrm{a}$ & $1.36 \mathrm{a}$ \\
\hline $\mathrm{CV} \%$ & 12.9 & 12.7 & 27.2 & 18.5 & 45.5 & 30.3 & 29.9 & 31.7 \\
\hline \multicolumn{9}{|c|}{120 days $^{1}$} \\
\hline Red net 50\% & $65.5 \mathrm{ab}$ & $1.00 \mathrm{a}$ & $12.0 \mathrm{a}$ & 295.16 a & $6.07 \mathrm{a}$ & $6.34 \mathrm{a}$ & $4.05 \mathrm{a}$ & $2.28 \mathrm{a}$ \\
\hline Blue net $50 \%$ & $71.4 \mathrm{ab}$ & 0.99 a & 13.8 a & $195.34 \mathrm{a}$ & 4.39 a & 5.36 a & $3.73 \mathrm{a}$ & $1.63 \mathrm{a}$ \\
\hline Black net $70 \%$ & $82.4 \mathrm{a}$ & $1.04 \mathrm{a}$ & $14.0 \mathrm{a}$ & 293.34 a & 3.62 a & $5.12 \mathrm{a}$ & $3.34 \mathrm{a}$ & $1.78 \mathrm{a}$ \\
\hline Black net $50 \%$ & $62.7 \mathrm{ab}$ & $1.01 \mathrm{a}$ & $11.8 \mathrm{a}$ & $274.53 \mathrm{a}$ & $7.22 \mathrm{a}$ & 6.92 a & $4.42 \mathrm{a}$ & $2.50 \mathrm{a}$ \\
\hline Black net $30 \%$ & $69.8 \mathrm{ab}$ & $1.04 \mathrm{a}$ & 14.0 a & 262.13 a & 5.04 a & 5.46 a & 3.88 a & $1.58 \mathrm{a}$ \\
\hline Full sun & $53.4 \mathrm{~b}$ & 0.98 а & $12.3 \mathrm{a}$ & $194.30 \mathrm{a}$ & 6.12 a & $7.06 \mathrm{a}$ & $4.71 \mathrm{a}$ & $2.35 \mathrm{a}$ \\
\hline CV\% & 14.2 & 16.5 & 27.4 & 42.0 & 36.4 & 37.8 & 36.6 & 45.6 \\
\hline \multicolumn{9}{|c|}{160 days $^{1}$} \\
\hline Red net 50\% & $77.2 \mathrm{~b}$ & $1.23 \mathrm{a}$ & $9.8 \mathrm{a}$ & $293.08 \mathrm{ab}$ & $14.56 \mathrm{a}$ & $9.04 \mathrm{a}$ & $6.21 \mathrm{a}$ & $2.83 \mathrm{a}$ \\
\hline Blue net $50 \%$ & $72.4 \mathrm{bc}$ & $1.26 \mathrm{a}$ & $11.5 \mathrm{a}$ & 229.09 b & 10.39 a & 7.29 a & 5.35 a & $1.94 \mathrm{a}$ \\
\hline Black net $70 \%$ & 90.0 a & $1.16 \mathrm{a}$ & $12.3 \mathrm{a}$ & 416.37 a & 10.36 a & 9.37 a & 6.78 a & 2.59 a \\
\hline Black net $50 \%$ & $64.0 \mathrm{c}$ & 1.39 a & 12.8 a & $280.62 \mathrm{ab}$ & 13.52 a & 8.72 a & 6.06 a & $2.68 \mathrm{a}$ \\
\hline Black net $30 \%$ & 68.1 bc & $1.23 \mathrm{a}$ & $11.0 \mathrm{a}$ & $243.90 \mathrm{~b}$ & 13.12 a & 8.02 a & $5.94 \mathrm{a}$ & 2.08 a \\
\hline Full sun & $53.6 \mathrm{~d}$ & $1.24 \mathrm{a}$ & $11.3 \mathrm{a}$ & $190.69 \mathrm{~b}$ & $21.71 \mathrm{a}$ & $7.73 \mathrm{a}$ & 5.46 a & $2.27 \mathrm{a}$ \\
\hline CV\% & 6.5 & 9.5 & 28.3 & 25.2 & 63.9 & 20.4 & 20.8 & 27.6 \\
\hline
\end{tabular}

${ }^{1}$ Means followed by the same letter in the column do not differ from each other by the Tukey test at $5 \%$ significance level.

${ }^{1}$ Médias seguidas pela mesma letra na coluna não diferem entre si pelo teste de Tukey a $5 \%$ de significância.

was highlighted with the highest value, such treatment was statistically different only from the black net $30 \%$ and full sun. On the other hand, the black net 30\% was only different from black net $70 \%$, reaching the same results, for instance, as the full sun treatment, which had the lowest values for stem length. This also happened with leaf area, as all net treatments were statistically equal, but only the black net $70 \%$ was different from full sun.
The variables stem diameter and leaf number did not present significant differences among treatments for all evaluation periods (Table 2), as it also happened for B. variegata.

At 160 days, the dry matter of roots had the highest values under the black net $50 \%$, black net $30 \%$, and full sun (Table 2) and was the only B. variegata var. candida variable that showed different results from

Revista Árvore, Viçosa-MG, v.38, n.6, p.1133-1145, 2014 
Table 2 - Stem length (SL), stem diameter (SD), leaf number (LN), leaf area (LA), and dry matter of roots (DMR), aerial part (DMAP), stem (DMS), and leaves (DML) of Bauhinia variegata var. candida seedlings cultivated under different light conditions along 160 days (2011).

Tabela 2 - Comprimento de caule (SL), diâmetro de caule (SD), número de folhas (LN), área foliar (LA) e matéria seca de raízes (DMR), parte aérea (DMAP), caule (DMS) e folhas (DML) de mudas de Bauhinia variegata var. candida cultivadas sob diferentes condições de luminosidade ao longo de 160 dias (2011).

\begin{tabular}{|c|c|c|c|c|c|c|c|c|}
\hline \multicolumn{9}{|c|}{ Bauhinia variegata var. candida } \\
\hline Light conditions & $\mathrm{SL}(\mathrm{cm})$ & $\mathrm{SD}(\mathrm{cm})$ & $\mathrm{LN}$ & $\mathrm{LA}\left(\mathrm{cm}^{2}\right)$ & $\operatorname{DMR}(\mathrm{g})$ & DMAP (g) & DMS (g) & DML (g) \\
\hline \multicolumn{9}{|c|}{40 days $^{1}$} \\
\hline Red net 50\% & $45.5 \mathrm{a}$ & 0.55 a & $11.8 \mathrm{a}$ & $236.55 \mathrm{ab}$ & $2.22 \mathrm{a}$ & $1.81 \mathrm{a}$ & 0.92 a & 0.89 a \\
\hline Blue net $50 \%$ & $42.3 \mathrm{ab}$ & 0.58 а & $11.5 \mathrm{a}$ & $193.79 \mathrm{ab}$ & $1.07 \mathrm{ab}$ & $1.58 \mathrm{a}$ & $0.70 \mathrm{a}$ & 0.88 a \\
\hline Black net $70 \%$ & 48.6 a & $0.45 \mathrm{a}$ & 10.8 а & $250.92 a b$ & $0.31 \mathrm{~b}$ & $1.24 \mathrm{a}$ & $0.55 \mathrm{a}$ & 0.69 а \\
\hline Black net $50 \%$ & 44.8 a & $0.58 \mathrm{a}$ & $11.8 \mathrm{a}$ & 299.52 a & $0.71 \mathrm{ab}$ & $1.89 \mathrm{a}$ & $0.87 \mathrm{a}$ & $1.02 \mathrm{a}$ \\
\hline Black net $30 \%$ & 37.6 ab & $0.46 \mathrm{a}$ & $9.8 \mathrm{a}$ & $199.07 \mathrm{ab}$ & $0.64 \mathrm{ab}$ & $1.40 \mathrm{a}$ & $0.66 \mathrm{a}$ & $0.74 \mathrm{a}$ \\
\hline Full sun & $32.6 \mathrm{~b}$ & 0.53 a & 10.5 a & $131.13 \mathrm{~b}$ & $1.18 \mathrm{ab}$ & $1.45 \mathrm{a}$ & $0.83 \mathrm{a}$ & 0.62 a \\
\hline CV\% & 11.9 & 13.9 & 12.3 & 30.4 & 76.1 & 27.8 & 27.6 & 29.7 \\
\hline \multicolumn{9}{|c|}{80 days $^{1}$} \\
\hline Red net 50\% & $58.4 \mathrm{ab}$ & $1.05 \mathrm{a}$ & $11.5 \mathrm{a}$ & $31.11 \mathrm{ab}$ & $2.75 \mathrm{a}$ & $4.55 \mathrm{a}$ & $2.56 \mathrm{a}$ & $2.01 \mathrm{a}$ \\
\hline Blue net $50 \%$ & $60.0 \mathrm{ab}$ & $0.91 \mathrm{a}$ & 13.8 a & $255.67 \mathrm{ab}$ & $2.76 \mathrm{a}$ & $4.02 \mathrm{a}$ & $2.21 \mathrm{a}$ & $1.81 \mathrm{a}$ \\
\hline Black net $70 \%$ & 75.9 а & 0.85 a & 16.5 a & $372.03 \mathrm{a}$ & $1.23 \mathrm{a}$ & $3.87 \mathrm{a}$ & $1.97 \mathrm{a}$ & $1.90 \mathrm{a}$ \\
\hline Black net $50 \%$ & $55.2 \mathrm{ab}$ & 0.85 a & 12.8 a & $232.46 \mathrm{ab}$ & $3.17 \mathrm{a}$ & $3.41 \mathrm{a}$ & $1.79 \mathrm{a}$ & $1.63 \mathrm{a}$ \\
\hline Black net $30 \%$ & $59.3 \mathrm{ab}$ & $0.83 \mathrm{a}$ & 15.0 a & $267.69 \mathrm{ab}$ & $2.13 \mathrm{a}$ & 5.58 а & $2.85 \mathrm{a}$ & 2.73 a \\
\hline Full sun & $45.5 \mathrm{~b}$ & 0.80 a & 12.3 a & $140.20 \mathrm{~b}$ & $2.74 \mathrm{a}$ & $3.21 \mathrm{a}$ & $1.76 \mathrm{a}$ & $1.46 \mathrm{a}$ \\
\hline CV\% & 22.3 & 13.4 & 22.4 & 35.0 & 61.8 & 41.4 & 38.4 & 46.2 \\
\hline \multicolumn{9}{|c|}{120 days $^{1}$} \\
\hline Red net $50 \%$ & 73.9 a & $1.09 \mathrm{a}$ & $11.8 \mathrm{a}$ & 241.99 a & $7.94 \mathrm{a}$ & 7.66 a & $5.12 \mathrm{a}$ & $2.55 \mathrm{a}$ \\
\hline Blue net $50 \%$ & $60.2 \mathrm{ab}$ & $1.05 \mathrm{a}$ & $12.3 \mathrm{a}$ & 290.27 a & 8.85 a & $7.24 \mathrm{a}$ & $4.47 \mathrm{a}$ & 2.77 a \\
\hline Black net $70 \%$ & $76.1 \mathrm{a}$ & $1.03 \mathrm{a}$ & $14.5 \mathrm{a}$ & 306.60 a & $3.71 \mathrm{a}$ & $6.10 \mathrm{a}$ & $4.01 \mathrm{a}$ & 2.09 a \\
\hline Black net $50 \%$ & $59.1 \mathrm{ab}$ & $1.03 \mathrm{a}$ & $10.5 \mathrm{a}$ & $254.74 \mathrm{a}$ & 7.62 a & $6.90 \mathrm{a}$ & $4.44 \mathrm{a}$ & $2.46 \mathrm{a}$ \\
\hline Black net $30 \%$ & $63.6 \mathrm{ab}$ & $1.13 \mathrm{a}$ & $13.0 \mathrm{a}$ & $222.39 \mathrm{a}$ & $5.50 \mathrm{a}$ & $7.61 \mathrm{a}$ & $5.03 \mathrm{a}$ & $2.58 \mathrm{a}$ \\
\hline Full sun & $47.9 \mathrm{~b}$ & $0.91 \mathrm{a}$ & $10.0 \mathrm{a}$ & $131.91 \mathrm{a}$ & 5.15 a & 4.96 a & 3.25 a & $1.70 \mathrm{a}$ \\
\hline CV\% & 13.0 & 14.8 & 25.0 & 53.8 & 39.7 & 29.9 & 27.1 & 44.4 \\
\hline \multicolumn{9}{|c|}{160 days $^{1}$} \\
\hline Red net 50\% & $75.1 \mathrm{ab}$ & $1.19 \mathrm{a}$ & $12.0 \mathrm{a}$ & $235.01 \mathrm{ab}$ & $9.58 \mathrm{~b}$ & 7.78 a & $5.57 \mathrm{a}$ & $2.20 \mathrm{a}$ \\
\hline Blue net $50 \%$ & $67.8 \mathrm{ab}$ & $1.15 \mathrm{a}$ & $11.3 \mathrm{a}$ & $274.23 \mathrm{ab}$ & $9.68 \mathrm{~b}$ & 7.50 а & 5.54 a & 2.21 a \\
\hline Black net $70 \%$ & 79.6 a & $1.19 \mathrm{a}$ & $12.3 \mathrm{a}$ & 342.62 a & $10.64 \mathrm{~b}$ & 8.13 a & $5.60 \mathrm{a}$ & 2.53 a \\
\hline Black net $50 \%$ & $68.0 \mathrm{ab}$ & $1.25 \mathrm{a}$ & $11.0 \mathrm{a}$ & $240.45 \mathrm{ab}$ & $13.10 \mathrm{ab}$ & 8.70 a & 6.08 a & 2.63 a \\
\hline Black net $30 \%$ & 65.3 bc & $1.16 \mathrm{a}$ & 9.5 a & $246.09 \mathrm{ab}$ & $17.48 \mathrm{a}$ & 9.38 а & $6.34 \mathrm{a}$ & $3.03 \mathrm{a}$ \\
\hline$\underline{\text { Full sun }}$ & $53.6 \mathrm{c}$ & $1.19 \mathrm{a}$ & $10.0 \mathrm{a}$ & $183.27 \mathrm{~b}$ & $13.68 \mathrm{ab}$ & $9.13 \mathrm{a}$ & $6.34 \mathrm{a}$ & $2.79 \mathrm{a}$ \\
\hline CV\% & 7.9 & 14.0 & 13.6 & 27.3 & 24.2 & 20.7 & 23.1 & 28.6 \\
\hline
\end{tabular}

${ }^{1}$ Means followed by the same letter in the column do not differ from each other by the Tukey test at $5 \%$ significance level.

${ }^{1}$ Médias seguidas pela mesma letra na coluna não diferem entre si pelo teste de Tukey a $5 \%$ de significância.

B. variegata ones. However, only the black net $30 \%$, which promoted the shortest stems, was statistically different from the other treatments.

Polynomial regression analyses for both $B$. variegata and $B$. variegata var. candida resulted in linear adjustments for stem length, stem diameter, and dry matter of roots, aerial part, stem, and leaves for all treatments (Figures 1 and 2), showing constantly increasing values among treatments along the experimental period. There were quadratic adjustments for leaf number, for both species and all treatments, with maximum values obtained between 74 and 110 days; the black net $70 \%$ was highlighted with the greatest numbers: B. variegata had 15.6 leaves between 91 and 99 days, while $B$. variegata var. candida presented 15.1 leaves between 92 and 105 days. The exception was for B. variegata var. candida, which red net 50\% 

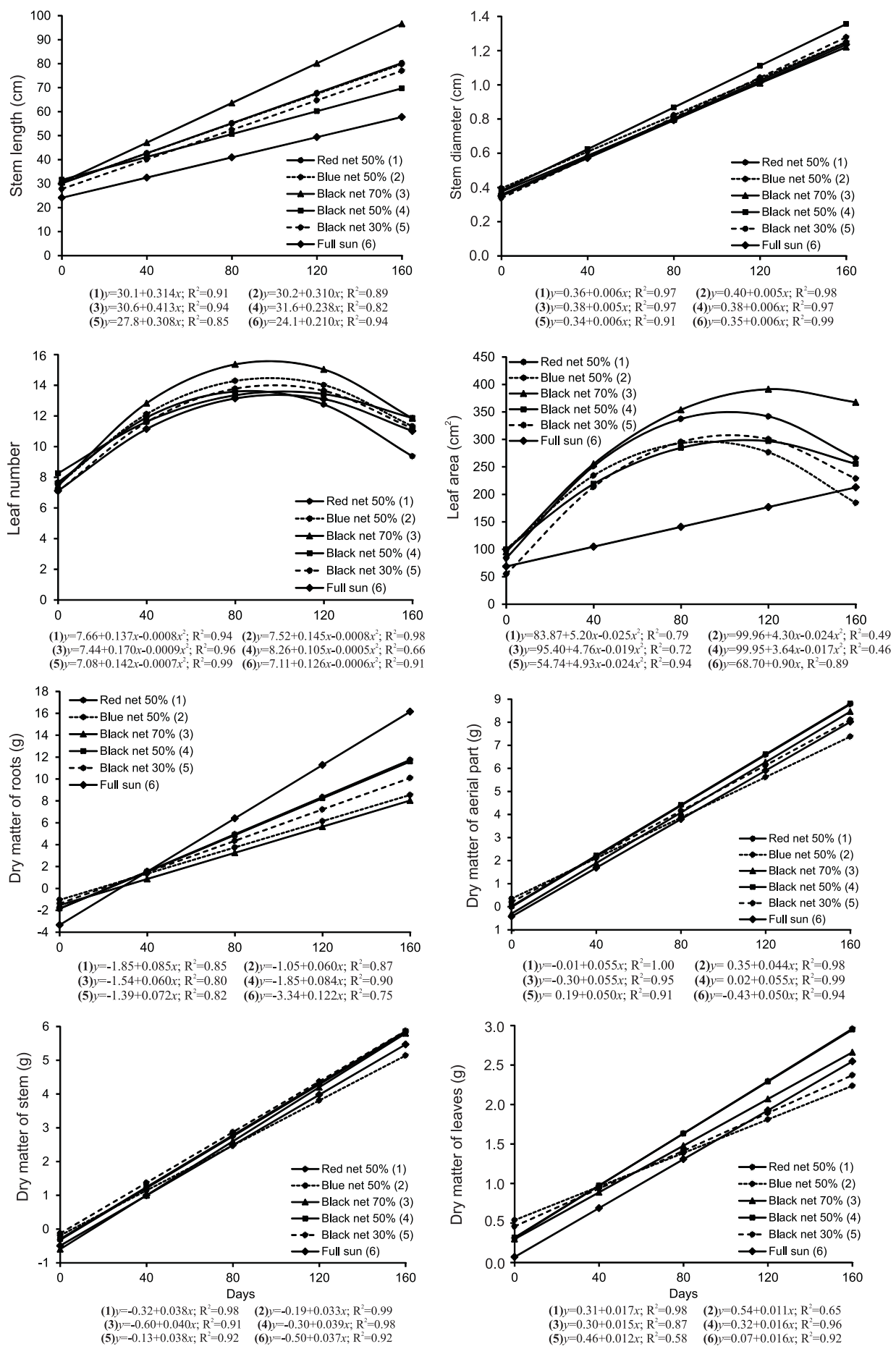

Figure 1 - Variations of stem length and diameter; leaf number and area; and dry matter of roots, aerial part, stem, and leaves of Bauhinia variegata seedlings cultivated under different light conditions along 160 days (2011).

Figura 1 - Variações de comprimento e diâmetro de caule, número de folhas, área foliar e matéria seca de raízes, parte aérea, caule e folhas de mudas de Bauhinia variegata cultivadas sob diferentes condições de luminosidade, ao longo de 160 dias (2011).

Revista Árvore, Viçosa-MG, v.38, n.6, p.1133-1145, 2014 

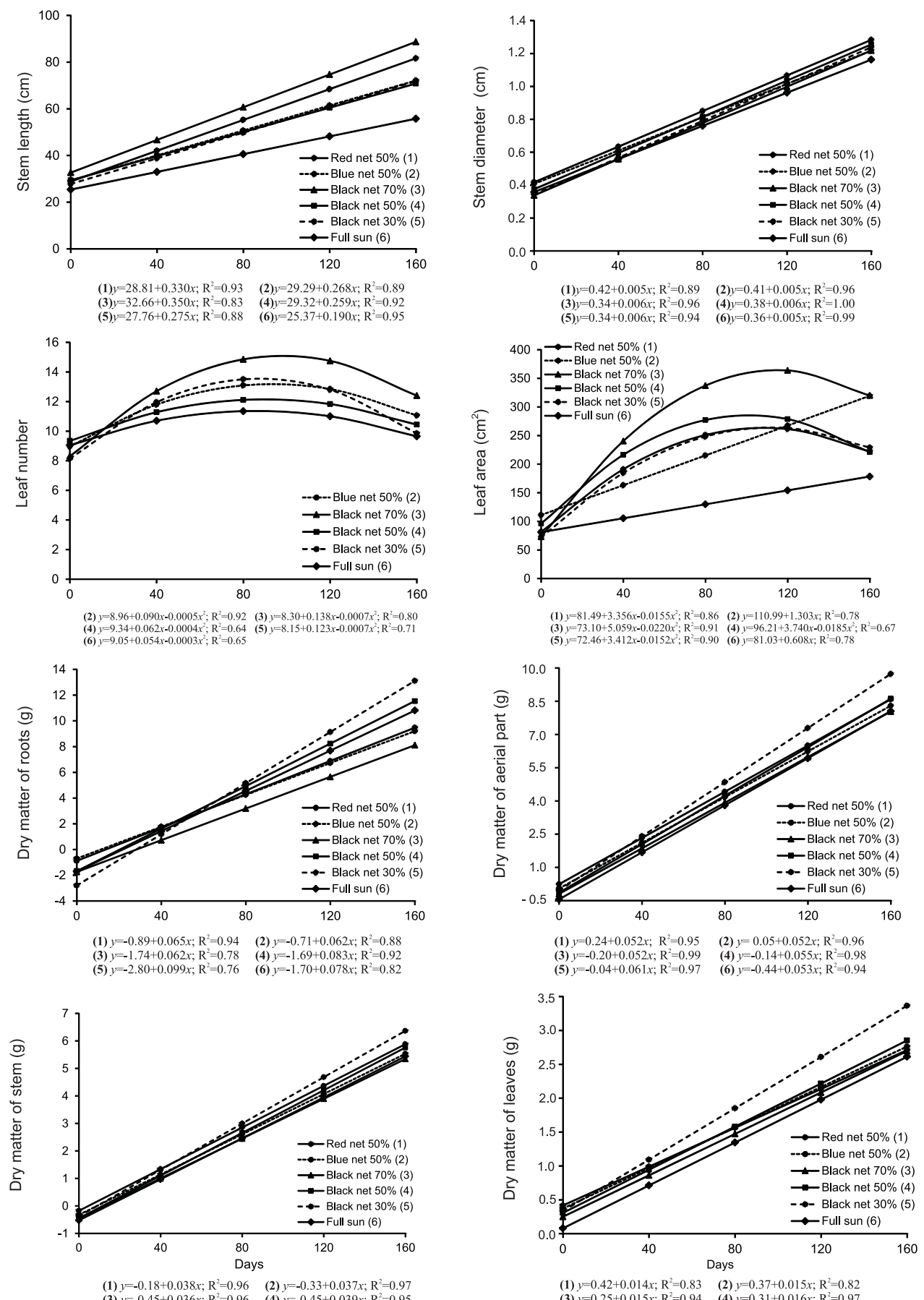

$\begin{array}{lll}\text { (1) } y=-0.18+0.038 x ; \mathbf{R}^{2}=0.96 & \text { (2) } y=-0.33+0.037 x ; \mathbf{R}^{2}=0.97\end{array}$ $\begin{array}{lll}\text { (3) } y=-0.45+0.036 x ; \mathbf{R}^{2}=0.96 & \text { (4) } y=-0.45+0.039 x ; \mathbf{R}^{2}=0.95 \\ \text { (5) } y=-0.38+0.042 x ; \mathbf{R}^{2}=0.97 & \text { (6) } y=-0.52+0.037 x ; \mathbf{R}^{2}=0.91\end{array}$ $\begin{array}{lll}\text { 3) } y=0.25+0.015 x ; \mathrm{R}^{2}=0.94 & \text { (4) } y=0.31+0.016 x ; \mathrm{R}^{2}=0.97 \\ \text { (5) } y=0.34+0.019 ; & \mathrm{R}^{2}=0.85 & \text { (6) } y=0.08+0.016 x ; \mathrm{R}^{2}=0.97\end{array}$

Figure 2 - Variations of stem length and diameter; leaf number and area; and dry matter of roots, aerial part, stem, and leaves of Bauhinia variegata var. candida seedlings cultivated under different light conditions along 160 days (2011).

Figura 2 - Variações de comprimento e diâmetro de caule, número de folhas, área foliar e matéria seca de raízes, parte aérea, caule e folhas de mudas de Bauhinia variegata var. candida cultivadas sob diferentes condições de luminosidade, ao longo de 160 dias (2011). 
regression was not significant and, therefore, was not represented. For both $B$. variegata and $B$. variegata var. candida, there were quadratic adjustments for leaf area for all net treatments, reaching maximum values between 91 and 125 days, and 101 and 115 days, respectively. The exceptions were the full sun for both species, and blue net $50 \%$ for $B$. variegata var. candida that resulted in linear adjustments. The best treatment was again the black net $70 \%$, which promoted $391.65 \mathrm{~cm}^{2}$ leaf area at 124 days for $B$. variegata, and $364.23 \mathrm{~cm}^{2}$ leaf area at 115 days for $B$. variegata var. candida.

Analysis of absolute growth rate of roots (AGR) of $B$. variegata seedlings, calculated separately for each treatment, showed that there were increasing values along time for the red net 50\% (0.027 to $0.212 \mathrm{~g} \mathrm{day}^{-1}$ ), black net $70 \%$ (0.007 to $\left.0.169 \mathrm{~g} \mathrm{day}^{-1}\right)$, and black net $50 \%$ ( 0.014 to $\left.0.158 \mathrm{~g} \mathrm{day}^{-1}\right)$. Seedlings of $B$. variegata var. candida presented raising values for all black nets: $30 \%$ ( 0.015 to 0.300 g day $\left.^{-1}\right), 50 \%$ (0.017 to 0.137 g day $\left.^{-1}\right)$, and $70 \%$ (0.007 to $\left.0.173 \mathrm{~g} \mathrm{day}^{-1}\right)$, and, also, full sun $\left(0.029\right.$ to $\left.0.213 \mathrm{~g} \mathrm{day}^{-1}\right)$. The greatest final increment for this species was promoted by the black net $30 \%$, what was previously indicated by both Tukey test (Table 2) and regression (Figure 2). For B. variegata, full sun was the treatment that promoted the greatest root growth at 160 days $\left(0.390 \mathrm{~g} \mathrm{day}^{-1}\right)$, although plants have shown a small variation before that period.

Results from leaf area ratio (LAR) and leaf weight ratio (LWR) (Figure 3) showed that seedlings of both species used increasingly smaller leaf area over time to produce the same dry matter amount. Although all treatments presented very close LAR values at the end of the experiment, those plants cultivated under full sun were more efficient, as they used the smallest leaf area to produce $1 \mathrm{~g}$ of dry matter. B. variegata seedlings cultivated under full sun, for instance, used only $6.48 \mathrm{~cm}^{2}$ leaf area, while $B$. variegata var. candida ones used $8 \mathrm{~cm}^{2}$. The black net $70 \%$ promoted the lowest plant efficiency among treatments, resulting in higher
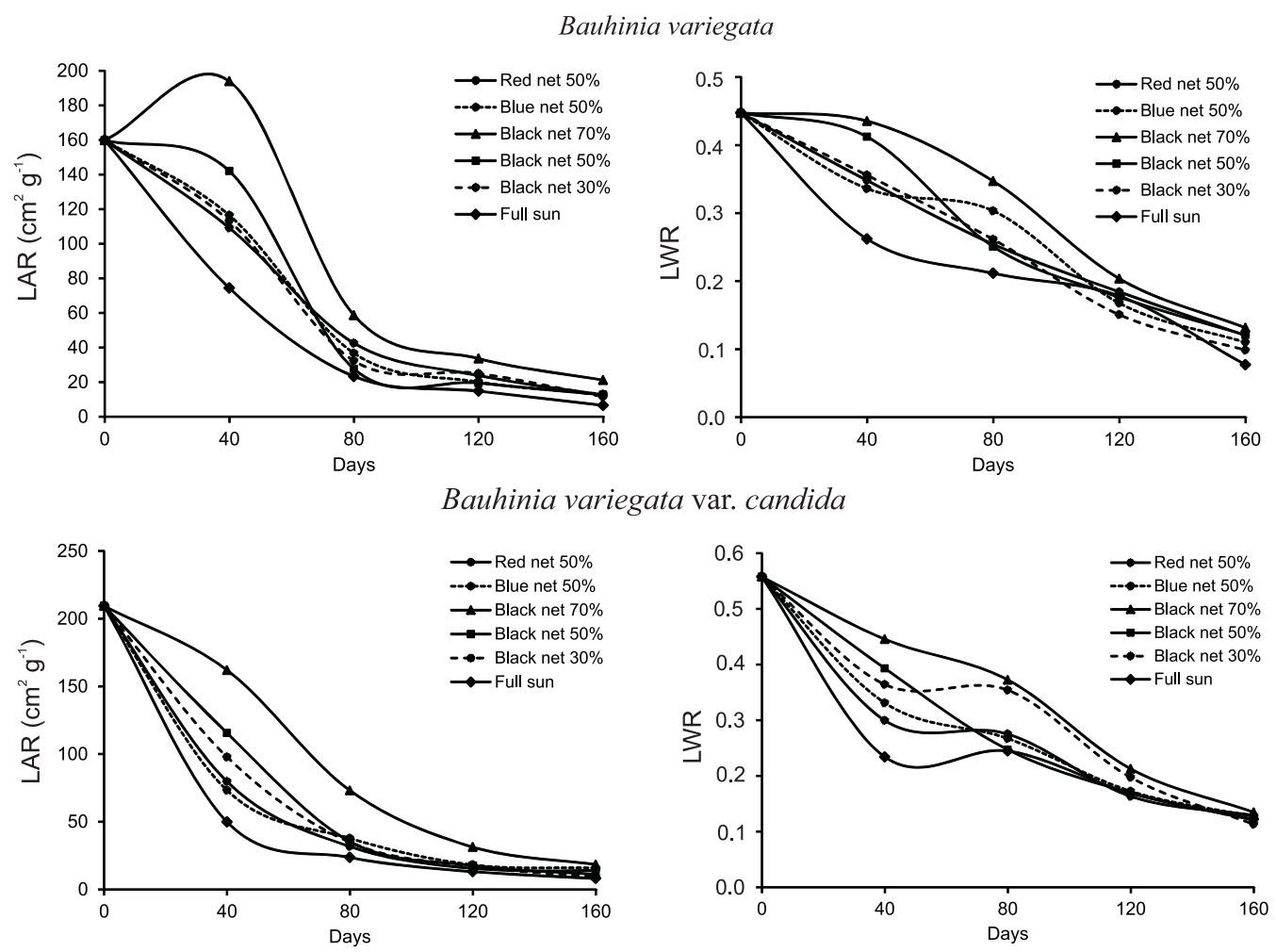

Figure 3 - Leaf area ratio (LAR) and leaf weight ratio (LWR) of Bauhinia variegata and B. variegata var. candida seedlings cultivated under different light conditions along 160 days (2011).

Figura 3 - Razão de área foliar (LAR) e razão de peso de folha (LWR) de mudas de Bauhinia variegata e B. variegata var. candida cultivadas sob diferentes condições de luminosidade, ao longo de 160 dias (2011).

Revista Árvore, Viçosa-MG, v.38, n.6, p.1133-1145, 2014 
LAR values for both species, as seedlings of $B$. variegata and $B$. variegata var. candida, used, respectively, 21.1 and $18.3 \mathrm{~cm}^{2}$ leaf area to produce the same amount of dry matter.

Values of leaf weight ratio (LWR), for both species, also showed that, by the end of the experiment, those plants cultivated under full sun maintained the fewest amount of dry matter in their leaves (Figure 3). Plants cultivated under the black net $70 \%$ stored the greatest amount of dry matter in their leaves along the experimental period, as the leaf area ratio (LAR) also indicates.

\section{DISCUSSION}

Plants of $B$. variegata cultivated under the black net $70 \%$ were the tallest of the experiment, indicating that those seedlings may have suffered stem elongation due to low luminosity as a way to escape light deficit. According to Benincasa (2003) and Meleiro and Graziano (2007), this characterizes the etiolation phenomenon since this species is, normally, cultivated under full sun (RORIZ, 2000; GLOBAL..., 2003) and would not be able to tolerate low light intensities (SCALON et al., 2003; MELEIRO; GRAZIANO, 2007). Rego and Possamai (2006) also mention that the ability of fast growth under shaded environments is an important mechanism of plant adaptation. Such statement is confirmed when the dry matter data, obtained from the black net $70 \%$, are considered, as they were statistically equal to the other treatments, what proves that other evaluated variables did not grow as fast as the stem. Furthermore, a poor root growth negatively influences seedling future cultivation, since plants with too long stems must have proportionally vigorous roots to anchor themselves properly in the soil.

Plants of Bauhinia forficata, Betula alleghanensis, and Bombacopsis glabra also presented lower growth when cultivated under full sun (GORDON, 1969; ATROCH et al., 2001; SCALON et al., 2003). However, each species seems to have a particular adaptation ability that varies according to the available luminosity (BOARDMAN, 1977; ALMEIDA et al., 2005). Eugenia uniflora seedlings, for instance, showed greater initial growth when cultivated under full sun (MARTINAZZO et al., 2007), while plants of Hymenaea courbaril var. stilbocarpa and Acacia mangium equally grew regardless several shading conditions (ALMEIDA et al., 2005).
Most treatments, for both species, promoted variation on leaf number and area along the experimental period (Figures 1 and 2). According to Reich et al. (2003), leaves may be considered a plant functional trait, as they significantly influence plant establishment, survival, and fitness. As so, Reich et al. (2003) state that functional traits, typically, present wide variations according to light/shade intensity, water availability, nutrient absorption, and temperature. Also, as perennial plants, Bauhinia species should really present a natural variation on leaf number and area as plants grow, new leaves are formed, and older leaves go through the senescence process. Such variation, with maximum and minimum values, would possibly be regularly repeated if the experimental period was longer.

Sun plants cultivated under shaded conditions tend to develop larger leaves with greater chlorophyll content as a way to compensate low luminosities (BOARDMAN, 1977; ENGEL; POGGIANI, 1991; ATROCH et al., 2001; ALMEIDA et al., 2005; REGO; POSSAMAI, 2006; MARTINAZZO et al., 2007; MIRALLES et al., 2011), although these leaves are usually thinner. Such feature may often turn these shaded leaves into more photosynthetically efficient leaves than those maintained under full sun (BOARDMAN, 1977; REGO; POSSAMAI, 2006; SOUZA et al., 2010).

In this sense, chlorophyll content is directly related to photosynthetic efficiency, thus, to plant growth and adaptation to different kinds of environments (ENGEL; POGGIANI, 1991; SCALON et al., 2003). As leaves are important photosynthetic organs that act on biological production, leaf area can be considered an important productivity index (SCALON et al., 2003) on seedling formation. This is the case of $B$. variegata and $B$. variegata var. candida, as their flowers, that are the final objective of the cultivation of these species, are produced as fast as two years from their germination, what requires an appropriate level of nutrient reserves.

Regarding $B$. variegata seedlings, the absence of statistical difference at the end of the experimental period for all dry matter variables shows that matter increment was not affected by the different nets. Consequently, the photosynthesis process was not affected either, once it is responsible for dry matter deposition which, in turn, is directly related to plant growth (ENGEL; POGGIANI, 1991).

Revista Árvore, Viçosa-MG, v.38, n.6, p.1133-1145, 2014 
Statistical similarity among treatments for stem diameter and leaf number, for both species, indicated that plants cultivated under full sun invested the greatest part of their produced photoassimilated compounds in stem thickness over height (ATROCH et al., 2001). This indicates that, although it has promoted slower growth, its development was equal to plants cultivated under the other nets; even when the plants had shorter stems, the diameter was similar to those from plants cultivated in the other environments.

Concerning the root dry matter of $B$. variegata var. candida, the highest values, which were obtained from those plants cultivated under full sun, indicate that they prioritized root development over shoot growth. Furthermore, in the case of $B$. variegata, full sun was the treatment that showed the greatest root increment (AGR) towards the end of the experiment, demonstrating, once more, that plants cultivated under such condition prioritized root development over growth of the aerial part. In fact, Miralles et al. (2011) mention that the reduction in plant evaporative demand, because of shading, affects its water absorption needs, what possibly diminishes root growth.

With respect to leaf area ratio (LAR) values, Benincasa (2003) mentions that they tend to decrease according to plant growth. This happens because, as more leaves are produced, the upper ones shade the lower ones, what results in the decrease of the useful leaf area from a certain plant stage. Benincasa (2003) highlights, yet, that the decrease in LAR values is also related to the increase of light intensity, thus, to plant adaptation ability to different light conditions. Therefore, the leaf area needed for the production of $1 \mathrm{~g}$ of dry matter decreases, within certain limits, with the increase of the incident luminosity (BENINCASA, 2003), what was found in this study. Furthermore, according to Claussen (1996) and Souza et al. (2010), when LAR values obtained from plants cultivated under high radiation are low, the amount of plant material exposed to possible damage caused by such high radiation would also be low.

Castro et al. (2005) mention that, during its development, the leaf, which usually absorbed photoassimilates, starts to produce and translocate these compounds to other organs. However, according to Henrique et al. (2011), matter allocation due to variations on the incident light is not yet fully understood. Also, according to Benincasa (2003), LAR values can be confused with the real dry matter and, as a matter of fact, this is demonstrated by the data collected from such variable in this experiment (Tables 1 and 2).

Values of leaf weight ratio (LWR) (Figure 3) show that plants cultivated under full sun, of both species, spared greater amounts of photoassimilates to other organs over their own leaves. On the other hand, those grown under the black net $70 \%$ accumulated more dry matter in their leaves what, consequently, may negatively influence future development. According to Benincasa (2003), the efficiency of photoassimilate exportation from the leaves is higher as plants grow, which is of great importance for agricultural production. As plants cultivated under full sun had smaller leaves, it is possible to infer that they were the most efficient among all treatments. These plants also had thicker leaves as there was no statistical difference for dry matter of leaves among treatments over the experimental period for B. variegata var. candida and at 80 days for $B$. variegata up to the final evaluation (Tables 1 and 2), what demonstrates good adaptation ability of these species. In the long term, these plants will possibly form trees with more appropriate characteristics for their cultivation. Benincasa (2003) suggests that the photoassimilate exportation from leaves may be difficult because of low radiation, what possibly happened with the use of the black net $70 \%$.

When results of the Tukey test were considered in isolation, the red net $50 \%$ and black net $50 \%$, in a general way, were the treatments that promoted the best results for, respectively, $B$. variegata and $B$. variegata var. candida. Although higher means of stem length and leaf area have been observed from plants of both species cultivated under the black net $70 \%$, these values are probably related to the etiolation phenomenon, what does not confer good quality to seedlings.

The growth relationships showed, however, that $B$. variegata seedlings grown under full sun had greater and faster root growth and were more efficient on the use of their leaves. Although B. variegata var. candida seedlings cultivated under the black net $30 \%$ had greater root growth and dry matter increase, full sun plants were also more efficient on the use of their produced photoassimilates.

Seedlings of trees that are usually cultivated under full sun are generally produced under shade or halfshade conditions. Atroch et al. (2001), for instance, 
concluded that Bauhinia forficata seedlings presented greater development under $30 \%$ shading. Plants of both $B$. variegata and $B$. variegata var. candida, however, as they showed more efficiency on the initial development under full sun, besides greater root growth, may be more adapted to tolerate inappropriate environmental conditions during full development, such as drought or low humidity (GORDON, 1969). In fact, Claussen (1996) mentions that when more matter is spared towards root growth, plants will be able to absorb more water, tolerating conditions of high radiation and, consequently, high transpiration rates.

According to Oren-Shamir et al. (2001), Shahak et al. (2004a), and Stamps and Chandler (2008), red nets, in general, would confer great plant growth, vigor, development of stems and flowers, and production of bigger fruits; on the other hand, blue nets would promote dwarfism, inhibiting stem growth and fruit development. However, as demonstrated by this study, these effects are particular for each species that responds differently to each light condition.

\section{CONCLUSIONS}

Seedlings of Bauhinia variegata and B. variegata var. candida were more efficient on the use of their photoassimilates when cultivated under full sun; thus, such condition is more appropriate for seedling growth. Both species showed etiolation when cultivated under the black net with $70 \%$ shading; hence, such condition is not recommended.

\section{ACKNOWLEDGMENTS}

To the Coordination for the Improvement of Higher Level Personnel (CAPES) for granting a doctorate scholarship to the first author.

\section{REFERENCES}

AGUIAR, I. B.; FIGLIOLIA, M. B. Armazenamento de sementes liofilizadas de bauínia-rósea (Bauhinia variegata L. var. variegata). Revista Brasileira de Horticultura

Ornamental, v.2, n.2, p.33-40, 1996.

ALMEIDA, S. M. Z.; SOARES, A. M.; CASTRO, E. M.; VIEIRA, C. V.; GAJEGO, E. B. Alterações morfológicas e alocação de biomassa em plantas jovens de espécies florestais sob diferentes condições de sombreamento. Ciência Rural, v.35, n.1, p.62-68, 2005.

ATROCH, E. M. A. C.; SOARES, A. M.; ALVARENGA, A. A.; CASTRO, E. M.

Crescimento, teor de clorofilas, distribuição de biomassa e características anatômicas de plantas jovens de Bauhinia forficata Link submetidas à diferentes condições de sombreamento. Ciência e Agrotecnologia, v.25, n.4, p.853-862, 2001.

BENINCASA, M. M. P. Análise de crescimento de plantas (noções básicas). 2.ed. Jaboticabal: FUNEP, 2003. 41p.

BOARDMAN, N. K. Comparative photosynthesis of sun and shade plants. Annual Review of Plant Physiology, v.28, p.355-377, 1977.

CASTRO, P. R. C.; KLUGE, R. A.; PERES, L. E. P. Manual de fisiologia vegetal: teoria e prática. Piracicaba: Agronômica Ceres, 2005. 650p.

CLAUSSEN, J. W. Acclimation abilities of three tropical rainforest seedlings to an increase in light intensity. Forest Ecology and Management, v.80, p.245-255, 1996.

ENGEL, V. L.; POGGIANI, F. Estudo da concentração de clorofila nas folhas e seu espectro de absorção de luz em função do sombreamento em mudas de quatro espécies florestais nativas. Revista Brasileira de Fisiologia Vegetal, v.3, n.1, p.39-45, 1991.

GLOBAL BOOK PUBLISHING PTY LTD. Flora. Willoughby: 2003. CD ROM.

GORDON, J. C. Effect of shade on photosynthesis and dry weight distribution in yellow birch (Betula alleghaniensis Britton) seedlings. Ecology, v.50, n.5, p.924-927, 1969.

HENRIQUE, P. C.; ALVES, J. D.; DEUNER, S.; GOULART, P. F. P.; LIVRAMENTO, D. E. Aspectos fisiológicos do desenvolvimento de mudas de café cultivadas sob telas de diferentes colorações. Pesquisa Agropecuária Brasileira, v.46, n.5, p.458-465, 2011.

LAU, C. P. Y.; RAMSDEN, L.; SAUNDERS, R. M. K. Hybrid origin of "Bauhinia blakeana" (Leguminosae: Caesalpinioideae), inferred using morphological, reproductive, and molecular data. 
American Journal of Botany, v.92, n.3, p.525-533, 2005.

LI, K.-T.; SYVERTSEN, J. Young tree growth and leaf function of citrus seedlings under colored shade netting. In: ANNUAL INTERNATIONAL CONFERENCE OF THE AMERICAN SOCIETY FOR HORTICULTURAL SCIENCE, 10., 2006, New Orleans; HortScience, v.41, n.4, p.1022, 2006.

LOBOS, G. A.; RETAMALES, J. B.; HANCOCK, J. F.; FLORE, J. A.; ROMERO-BRAVO, S.; POZO, A.Del. Productivity and fruit quality of Vaccinium corymbosum cv. Elliott under photoselective shading nets. Scientia

Horticulturae, v.153, n.1, p.143-149, 2013.

MAK, C. Y.; CHEUNG, K. S.; YIP, P. Y.; KWAN, H. S. Molecular evidence for the hybrid origin of Bauhinia blakeana (Caesalpinioideae).

Journal of Integrative Plant Biology, v.50, n.1, p.111-118, 2008.

MARTINAZZO, E. G.; ANESE, S.;

WANDSCHEER, A. C. D.; PASTORINI, L. H. Efeito do sombreamento sobre o crescimento inicial e teor de clorofila foliar de Eugenia uniflora Linn (pitanga) - Família Myrtaceae. Revista Brasileira de Biociências, v.5, p.162-164, 2007.

MATHUR, J.; MUKUNTHAKUMAR, S. Micropropagation of Bauhinia variegata and Parkinsonia aculeata from nodal explants of mature trees. Plant Cell, Tissue and Organ Culture, v.28, p.119-121, 1992.

MELEIRO, M.; GRAZIANO, T. T.

Desenvolvimento de tapeinóquilo em diferentes condições de luminosidade. Revista

Brasileira de Horticultura

Ornamental, v.13, n.1, p.63-72, 2007.

MEIRELLES, A. J. A.; PAIVA, P. D. O.; OLIVEIRA, M. I.; TAVARES, T. S. Influência de diferentes sombreamentos e nutrição foliar no desenvolvimento de mudas de palmeira ráfia Rhapis excelsa (Thunberg) Henry ex. Rehder. Ciência e Agrotecnologia, v.31, n.6, p.1884-1887, 2007.

MIRALLES, J.; MARTÍNEZ-SÁNCHEZ, J. J.; FRANCO, J. A.; BAÑÓN, S. Rhamnus alaternus growth under four simulated shade environments: morphological, anatomical and physiological responses. Scientia Horticulturae, v.127, p.562-570, 2011.

OREN-SHAMIR, M.; GUSSAKOVSKY, E. E.; SHPIEGEL, E.; NISSIM-LEVI, A.; RATNER, K.; OVADIA, R.; GILLER, Y. E.; SHAHAK, Y. Coloured shade nets can improve the yield and quality of green decorative branches of Pittosporum variegatum. Journal of Horticultural Science \& Biotechnology, v.76, n.3, p.353-361, 2001.

RAIJ, B.; ANDRADE, J. C.; CANTARELLA, H.; QUAGGiO, J. A. (Ed.) Análise química para avaliação da fertilidade de solos tropicais. Campinas: Instituto Agronômico, 2001. 284p.

REGO, G. M.; POSSAMAI, E. Efeito do sombreamento sobre o teor de clorofila e crescimento inicial do jequitibá-rosa. Boletim de Pesquisa Florestal, n.53, p.179-194, 2006.

REICH, P. B.; WRIGHT, I. J.; CAVENDER-BARES, J.; CRAINE, J. M.; OLEKSYN, J.; WESTOBY, M.; WALTERS, M. B. The evolution of plant functional variation: traits, spectra, and strategies. International Journal of Plant Sciences, v.164, n.S3, p.S143-S164, 2003.

RORIZ, A. (Ed.) 1001 plantas \& flores. São Paulo: Europa, 2000. 258p.

SCALON, S. P. Q.; MUSSURY, R. M.; RIGONI, M. R.; SCALON FILHO, H. Crescimento inicial de mudas de Bombacopsis glabra (Pasq.) A. Robyns sob condição de sombreamento. Revista Árvore, v.27, n.6, p.753-758, 2003.

SHAHAK, Y.; GUSSAKOVSKY, E. E.; COHEN, Y.; LURIE, S.; STERN, R.; KFIR, S.; NAOR, A.; ATZMON, I.; DORON, I.; GREENBLAT-AVRON, Y. ColorNets: a new approach for light manipulation in fruit trees. Acta

Horticulturae, v.636, p.609-616, 2004a.

SHAHAK, Y.; GUSSAKOVSKY, E. E.; GAL, E.; GANELEVIN, R. ColorNets: crop protection and light-quality manipulation in one technology. 
Acta Horticulturae, v.659, p.143-151, 2004b.

SOUZA, G. S.; CASTRO, E. M.; SOARES, A. M.; PINTO, J. E. B. P. Características biométricas e fisiológicas de plantas jovens de Mikania glomerata Sprengel e Mikania laevigata Schultz Bip. ex Baker cultivadas sob malhas coloridas.
Revista Brasileira de Biociências, v.8, n.4, p.330-335, 2010.

STAMPS, R. H.; CHANDLER, A. L. Differential effects of colored shade nets on three cut foliage crops. Acta Horticulturae, v.770, p.169-176, 2008. 
